\title{
5. Die Anstalt als Bildungsstätte und kaufmännischer Betrieb
}

\subsection{Die Einrichtung von Arbeitsplätzen}

Ein Ziel der Stationsgründung war es, Arbeitsmöglichkeiten für Wissenschaftler zu schaffen. Dazu wurden, wie an der Zoologischen Station Neapel, sogenannte „Arbeitstische“ eingerichtet. Dies bedeutete, daß Arbeitsfläche und außerdem Geräte, Chemikalien und andere Verbrauchsmaterialien bereitgestellt wurden. Diese „Arbeitstische“ konnten von einzelnen deutschen Ländern bzw. dem Ausland gemietet werden. Ähnlich wie in Neapel standen pro Land und Jahr ein bis zwei Tische zur Verfügung. Über die Vergabe entschied das Kultusministerium des entsendenden Landes. Die Institutsdirektoren beantragten dort für ihre jeweiligen Mitarbeiter den Arbeitsplatz. Dann erfolgte die formale Zustimmung. Vorher oder gleichzeitig fragten die interessierten Wissenschaftler bzw. deren Vorgesetzte in der Anstalt an. Junge, unbekannte Wissenschaftler bzw. Studenten wurden durch ihre Vorgesetzten empfohlen. Interessierten sich mehrere Anwärter für einen Arbeitsplatz, wurde die Entscheidung in der Regel nach inhaltlichen Gesichtspunkten von der Anstalt getroffen. In den ersten Jahren befaßte sich Friedrich Heincke selbst damit, später wurde diese Aufgabe Mitarbeitern übertragen. So ist belegt, daß zunächst Clemens Hartlaub die Belegung der Arbeitstische koordinierte.

Leider sind im Gegensatz zur Zoologischen Station Neapel kaum Angaben über die Nutzung der Arbeitsplätze überliefert. Die wenigen vorhandenen Hinweise sind zufälligen Entdeckungen in Nachlässen zu verdanken. Einige allgemeine Bemerkungen sind dem Briefwechsel des Direktors mit dem Kultusministerium zu entnehmen.

So ist belegt, daß es in den ersten Jahren sehr schwierig war, die Tische zu besetzen. Zum Kummer von Heincke entwickelte sich die Nachfrage erst nach und nach. Besonders im ersten Jahr war das Ergebnis deprimierend - es bewarben sich nur acht Interessenten. Und dies, obwohl die Bedingungen sehr günstig waren: die Nutzung war kostenfrei, man hatte bis zu drei Monaten nur $10 \mathrm{M}$, später RM, an den Bibliotheksfonds zu zahlen, lediglich die verbrauchten Chemikalien mußten ersetzt werden.

Heincke warb auf Fachtagungen und in Vorträgen für die Arbeitsmöglichkeiten an der Station und zeigte öffentlich seine Verwunderung über die geringe Nachfrage. Er erinnerte daran, daß nicht nur einzelne Gelehrte, sondern auch Gremien, wie die Zoologische Gesellschaft und die Königlich-Preußische Akademie der Wissenschaften um Einrichtung einer solchen Institution gebeten hatten. Eine Ursache für die schlechte Belegung der Arbeitsplätze wurde in der Konkurrenz durch die Zoologische Station Neapel gesehen. So rief Verärgerung hervor, daß sich die Nachfrage an der Zoologischen Station Neapel ungleich günstiger entwickelte und sich - besonders nach Einrichtung einer physiologischen Abteilung in Neapel - vermehrt Ausländer bewarben. Auch die vier preußischen Arbeitstische in Neapel waren stets belegt. Vermutlich war dies - neben den steigenden Einnahmen, die aus dem Versand von Präparaten, Tieren und den Eintrittsgeldern für den Besuch des Aquariums erzielt wurden - eine Ursache dafür, daß Althoff die Förderung der Zoologischen Station Neapel durch das Reich in Frage stellte. Althoff sah in dieser Subventionierung durch das Reich einen unzulässigen Eingriff in Länderzuständigkeiten und drohte mit Kündigung der vier preußischen Tische. Er warf Dohrn vor, er bereichere sich aus Reichssubventionen (vgl. Patsch 1980). Eingefügt sei, daß Theodor Heuss ${ }^{160}$, der mit der Familie

160 Theodor Heuss (1884-1963) 
Dohrn eng befreundet war, das Verhalten Althoffs als Musterbeispiel von Ressortgeist und bürokratischer Enge bezeichnete (vgl. Heuss. 1948).

Nach und nach entwickelte sich auch die Nachfrage nach Arbeitstischen auf Helgoland, vermutlich dauerte es eine Weile, bis sich die guten Möglichkeiten herumgesprochen hatten. Folgende Tabelle gibt einen Überblick über die Belegung der Arbeitsplätze an der Anstalt auf Helgoland zwischen 1896-1934. Für die Jahre 1892-1896 existieren keine Zahlenangaben, bis 1906 wurden sie nicht jährlich aufgeschlüsselt. In die Tabelle aufgenommen wurden auch die Zahl der Teilnehmer an den Kursen, die ab 1920 durchgeführt wurden.

Tabelle 3. Beleger der Arbeitsplätze pro Jahr (zusammengestellt aus den Berichten der jeweiligen Direktoren der Anstalt)

\begin{tabular}{|lccc|}
\hline Jahr & Forscher & Kursteilnehmer & insgesamt \\
\hline $1896-1899$ & 14 & - & 14 \\
$1900-1903$ & 19 & - & 19 \\
$1903-1904$ & 19 & - & 19 \\
$1904-1906$ & 21 & - & 21 \\
1907 & 21 & - & 21 \\
1908 & 32 & - & 32 \\
1911 & 36 & - & 51 \\
1912 & $51(53)$ & - & $423 ?)$ \\
1913 & 42 & - & 5 \\
1914 & 5 & 28 & 16 \\
1919 & 16 & 81 & 76 \\
1920 & 48 & 67 & 130 \\
1921 & 49 & 96 & 153 \\
1922 & 86 & 100 & 180 \\
1923 & 90 & 140 & 206 \\
1924 & 106 & 64 & 216 \\
1925 & 76 & 132 & 142 \\
1926 & 78 & 172 & 259 \\
1927 & 127 & 244 & 277 \\
1928 & 105 & 184 & 364 \\
1929 & 120 & 189 & 301 \\
1930 & 17 & 144 & 303 \\
1931 & 114 & 85 & 257 \\
1932 & 113 & 155 & 87 \\
1933 & 102 & & 258 \\
1934 & 103 & & \\
& & & \\
+) differierende Angabe & & & \\
& & & \\
& & & \\
\hline
\end{tabular}

Ab Herbst 1896 konnten mehr Arbeitsplätze zur Verfügung gestellt werden, da das frühere Postgebäude zur Benutzung offen war. Die Zahl der Arbeitsplätze betrug nun sechs bis sieben. Es wurde aber, falls der Bedarf weiter ansteigen sollte, in Aussicht gestellt, daß die Anstalt die Ausrüstung von Arbeitsplätzen in Privatwohnungen übernehmen könne. Als dann ab 1910 - wie erhofft - die Nachfrage tatsächlich stieg, waren die Kapazitäten der Anstalt überfordert. So klagte Heincke im Jahresbericht 1912: 
„die Zahl der ambulanten Gelehrten, die . . Arbeitsplätze in der Anstalt benutzten, ...53 (betrug, d. V.) gegenüber $36 \mathrm{im}$ Vorjahre. Hiermit ist die höchste Zahl von Besuchern laut Bestehen der Anstalt erreicht. Zeitweilig konnten die Besucher nur dadurch plaziert werden, daß die Arbeitszimmer der wissenschaftlichen Beamten mit benutzt wurden“ (20).

Die erhöhte Nachfrage ist jedoch nicht nur durch das wachsende Interesse der Wissenschaftler zu erklären. So fällt auf, daß die Arbeitsplätze nicht nur von Wissenschaftlern belegt wurden. Wie aus einem privaten Brief des Physiologen und späteren Nobelpreisträgers Otto Meyerhof an seinen Kollegen Otto Warburg hervorgeht, nutzten viele arbeitslose Lehrer die Möglichkeit zur Weiterbildung an der Station. Im Jahre 1914 erreichte die Nachfrage einen Tiefstand, nur fünf Forscher fanden den Weg nach Helgoland. Schon der Sommer stand für die Arbeit nicht mehr zur Verfügung. Am 1. August begann der I. Weltkrieg. Die Station wurde für vier Jahre geschlossen. Erst im Sommer 1919 konnten wieder Gäste aufgenommen werden.

Es fällt auf, daß nach Beendigung des I. Weltkrieges die Zahl der Gastforscher wiederum sehr stark anstieg. Wie aus dem Schriftwechsel vergleichbarer Einrichtungen, zum Beispiel der Zoologischen Station Neapel, bekannt ist, konzentrierten sich deutsche Wissenschaftler nun an der einzigen deutschen Meeresstation, weil Aufenthalte an fremden Küsten aus Devisengründen abgesagt werden mußten. Auf Helgoland bemühte man sich inzwischen auf Grund der Devisenknappheit, gutzahlende Amerikaner und Schweizer als Gäste zu gewinnen. Um an konvertierbare Währung zu kommen, wurden auf Helgoland Wechselstuben eingerichtet. Es gab jedoch Vorbehalte, die man gegenüber Vertretern aus jenen Staaten hatte, die im Krieg auf der Seite der Gegner Deutschlands gestanden hatten. Dies betraf vor allem Engländer und Franzosen. Hier fanden besondere Abstimmungen zwischen dem Institutsdirektor und dem Ministerium statt.

Das steigende Interesse an der Arbeit auf der Station war aber auch durch die Verbesserung der Möglichkeiten bedingt, die die Anstalt ihren Nutzern bot. Das betraf sowohl die Hilfe, die durch das Personal der Anstalt gewährt wurde, als auch die technische Ausstattung. So standen 1896 für die Betreuung der Arbeitsplatzinhaber mehrere Angestellte der Biologischen Anstalt zur Verfügung, ein Fischmeister und drei Mitarbeiter. Außerdem waren zwei Diener gern bereit, die Forscher zu unterstützen. Der Präparator, vertraut mit der Anfertigung mikroskopischer Präparate und mit dem Mikrotom, konnte in besonderen Fällen hinzugezogen werden. Ein größeres Segelfahrzeug mit Petroleummotor („Augusta I") war mit Kurre und Dredgen ausgerüstet und konnte für Fischerei in größeren Tiefen genutzt werden. Außerdem gab es vier kleinere Boote, mit denen auch Exkursionen durchgeführt werden konnten. Ein Schauaquarium stand 1896 noch nicht zur Verfügung, wohl aber Einrichtungen zur Aufstellung kleiner Behälter, die belüftet werden konnten.

Die Bibliothek wurde ständig erweitert und umfaßte 1896 bereits 2500 Bände. Besonders umfangreich war der Bestand an faunistischer Literatur. $\mathrm{Zu}$ den vollständig vorhandenen Zeitschriften und größeren Werken gehörten „Report on the scientific results of the voyage of the H. M. S. Challenger", alle Publikationen der Zoologischen Station Neapel, die „Ergebnisse der Plankton-Expedition der Humboldt-Stiftung“, das „Archiv für Naturgeschichte“, das „Morphologische Jahrbuch“, „Zoologische Jahrbücher, Abt. für Allgemeine Zoologie und Physiologie der Tiere. Spengel.“, verschiedene Zeitschriften für wissenschaftliche Mikroskopie, Arbeiten aus dem Zoologischen Institut in Triest, das „Wilhelm Roux Archiv für Entwicklungsmechanik“, der „Anatomische Anzeiger“, der „Zoologische Anzeiger“, das „Zoologische Centralblatt“. Diese Bibliothek stand den auf Helgoland arbeitenden Wissenschaftlern zur Verfügung. Von allen auf Helgoland entstandenen Arbeiten wurde selbstverständlich ein Sonderdruck der Sammlung hinzugefügt. 
Bereits bei der Anmeldung zu Forschungsaufenthalten wurden die Wissenschaftler beraten, z. B. wurden ihnen Empfehlungen über den günstigsten Zeitpunkt gegeben. Forschern, die mit Tieren arbeiten wollten, die in geringer Tiefe auf dem breiten, Helgoland umgebenden Klippengürtel vorkommen, riet man, im Winter oder im Frühjahr auf die Insel zu kommen, weil dann die Ebben tiefer sind. Für Interessenten an pelagischen Organismen sei aber, so wurde betont, zu jeder Jahreszeit reiches Material vorhanden. Ansonsten riet man, wegen der Witterungsverhältnisse das Frühjahr oder den Sommer zu wählen.

Von der Anstalt wurden Übersichtsdarstellungen erarbeitet, die den Besuchern zur Orientierung über die auf Helgoland zur Verfügung stehenden biologischen Objekte empfohlen wurden. Auch über das Helgoländer Plankton wurde informiert. Die regelmäßig erscheinenden Larvenformen wurden besonders hervorgehoben.

Vor Ort eine preiswerte Unterkunft zu finden, war nicht so einfach. Aber auch hier waren Angestellte der Anstalt behilflich. Heincke selbst empfahl bei „gemäßigten Ansprüchen" gute, heizbare Wohnungen für 10-12 Mark bei dem zoologischen Fischer Hilmar Lührs oder bei Frau Payens, die die Pension „London Tavern“ führte. Bei Frau Payens konnte man in Vollpension wohnen, die Kosten schwankten zwischen 130 und 150 Mark pro Monat. Dort war es auch möglich, ein gutes Frühstück zu bekommen (1896 f. 60 Pfennige) bzw. einen Mittagstisch „ohne Weinzwang“ (1,50/2,- M).

Die Ausstattung der Arbeitsplätze für selbständige Gelehrte war, das ist alten Schilderungen zu entnehmen, von Anfang an modern und wurde in Abstimmung mit dem Kultusministerium stets verbessert und erweitert. Diese Veränderungen waren den Wünschen der Gelehrten und Kursteilnehmer angepaßt. Im Jahre 1893 war die Ausstattung der Tische korrespondierend zu den Arbeitsaufgaben der Anstalt auf morphologisch-anatomische Arbeiten ausgerichtet. Es standen zehn einfache Mikroskope zur Verfügung, sieben Präpariermikroskope, drei Mikrotome und eine "photographische Camera“. Außerdem gab es Ösen für Paraffineinbettung, ein anatomisches Besteck, zahlreiche Aärometer und Thermometer. Die Mehrzahl der Mikroskope und Immersionssysteme stammte aus der Werkstatt von R. Winkel, die Mikrotome wurden von der Firma A. Becker aus Göttingen bezogen. Die Kamera, „mikrophotographischer Apparat“ genannt, sowie ein größeres Stativ stammten von der Firma Steinheil. Ab Herbst 1896 wurde auch eine Dunkelkammer eingerichtet, so daß die Wissenschaftler ihre Fotos vor Ort selbst entwickeln konnten.

Eine wichtige Dienstleistung der Anstalt für die Wissenschaftler bestand in der Bereitstellung von lebendem Untersuchungsmaterial. Hierfür konnten die erwähnten Boote genutzt werden.

Trotz der Mühe, die sich die Angestellten gaben, blieb bei den Tischnutzern nicht immer ein erfreulicher Eindruck über den Aufenthalt in Helgoland zurück. Besonders Wissenschaftler, die die Neapler Station kannten, die wesentlich von der herausragenden Persönlichkeit Anton Dohrns bestimmt wurde, äußerten sich z. T. spöttisch. So verdanken wir Otto Meyerhof, der im Sommer 1912 auf Helgoland arbeitete, folgende ironische Schilderung:

„.. . das einzige noch am Leben befindliche Motorboot der Zoologischen Anstalt hat gerade gestern seine Kolbenstange gebrochen. Dies wie manche andre, z. B. das Fehlen von Gas und Elektrizität in der Anstalt, obwohl beides auf Helgoland vorrätig, ist auf den durch die Senilität des Direktors bewirkten Schlendrian zurückzuführen. Sonst aber, was Ausrüstung, Einrichtung, Platz etc. anbelangt, ist eigentlich alles recht nett und da ich gegenwärtig außer dem Direktor dort allein bin, auch sehr bequem und zugänglich für mich. Die Bibliothek ist biologisch (ausgenommen Physiologie) sehr reichhaltig, Apparatur für das meiste wenigstens in Einzahl da, so daß man Kjehldals, Gefrierbestimmungen, Winklertitrationen etc. machen könnte“(150). 


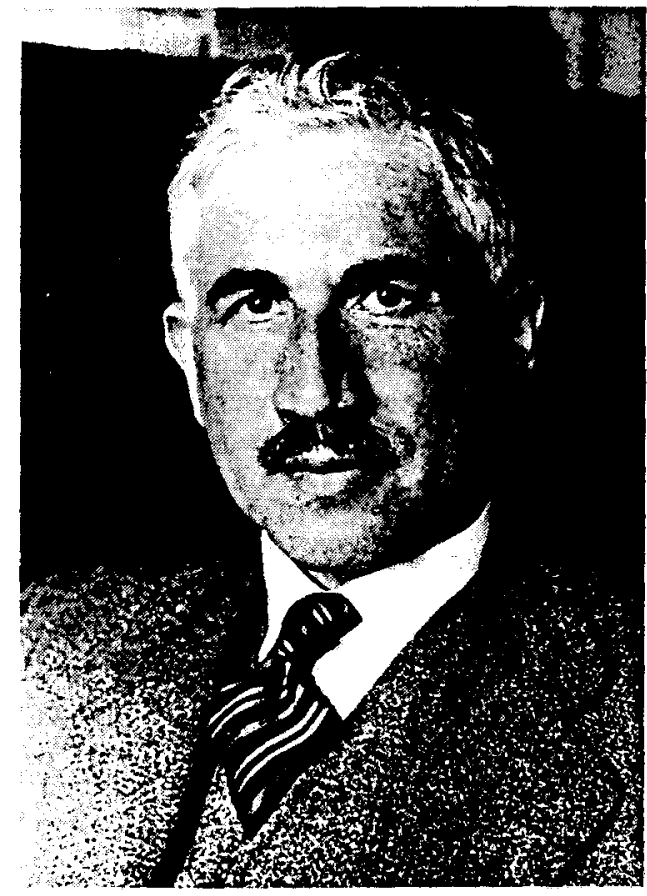

Abb. 40. Der Biochemiker Otto Meyerhof (1854-1951) erhielt 1922 den Nobelpreis. Er arbeitete 1912 auf Helgoland. Archiv der Akademie der Wissenschaften Berlin, Fotosammlung.

Angemerkt sei, daß der noch junge Meyerhof Heincke sehr subjektiv und ungerecht beurteilte. Heincke hatte sich intensiv bemüht, das Boot wieder reparieren bzw. durch ein besseres ersetzen zu lassen.

Allerdings fiel natürlich im Vergleich zu Neapel auf, daß die Möglichkeiten auf Helgoland schlechter waren. So existierte an der Zoologischen Station Neapel schon seit 1890 eine physiologische Abteilung. Ihr Fehlen auf Helgoland wurde von den Besuchern als großer Mangel empfunden. Offensichtlich wurden mehrere Anfragen an das Ministerium gerichtet, denn Heincke erklärte nach dem I. Weltkrieg in einem Schreiben an das Kultusministerium, daß die räumliche Beschränkung und der Geldmangel bisher die Einrichtung einer solchen Abteilung verhindert hätten. Er stellte einen entsprechenden Antrag. Bekannte deutsche Physiologen, unter ihnen Besucher der Anstait Helgoland, stützten das Anliegen durch ausführliche Gutachten. Heinckes Antrag wurde durch ein Gutachten vom Direktor des Physiologischen Instituts der Universität Rostock, Winterstein, flankiert. Auch Otto Meyerhof und der Physiologe Rudolf Höber ${ }^{161}$, von 1914 bis 1933 ordentlicher Professor für Physiologie und Direktor des Universitätsinstituts, halfen, indem sie eine „Denkschrift betreffend Einrichtung einer physiologischen Abteilung an der zoologischen Station in Helgoland" verfaßten. Beide Autoren begründeten die Notwendigkeit damit, daß die Ergebnisse der Physiologie nicht nur von Wert für die Physiologie des Menschen seien,

161 Rudolf Höber (1873-1953) 
sondern auch für die Pathologie, Fragen der Fischerei und andere Ernährungsprobleme. Sie erklärten ausführlich, welche großartigen wissenschaftlichen Leistungen in physiologischen Labors des Auslandes (z. B. in Neapel und Woods Hole) erbracht wurden, und betonten, daß die deutsche Wissenschaft nicht zurückstehen dürfe. Außerdem erläuterten sie die große Bedeutung für die Weiterarbeit der Wissenschaftler angesichts der angespannten Devisenlage Deutschlands und stellten in Aussicht, daß die Biologische Anstalt Helgoland ähnlich wie die Zoologische Station Neapel ein Zentrum des wissenschaftlichen und kulturellen Austauschs werden könne. Meyerhof hatte den Vorteil, persönliche Erfahrungen einbringen zu können:

„Auch hat einer von uns sich in eigener physiologischer Arbeit von der Möglichkeit überzeugt, auf Helgoland derartige physiologische Untersuchungen, wenn auch in beschränkterem Umfange als in Neapel, auszuführen. Allerdings müssen die Arbeitsplätze mit elektrischer Licht- und Kraftleitung versehen werden, eine Reihe allgemein notwendiger Apparate, Motoren, elektrische Zentrifuge, heizbare Wasserkästen (Thermostaten), elektrische Apparatur (Widerstände, Akkumulatoren, Stromanzeiger) und ein chemisches Laboratorium, das einfache Analysen gestattet“ (27).

Die Wissenschaftler schlugen die Einrichtung von zunächst vier Arbeitsplätzen vor, spezielle Arbeitsinstrumente sollte jeder Forscher selbst mitbringen. Otto Warburg, damals noch Leiter der Abteilung Physiologie im Kaiser-Wilhelm-Institut für Biologie in Dahlem, stellte eine Liste der für eine Erstausstattung notwendigen Geräte zusammen, die bei 55000 Mark abschloß. Hierzu gehörte neben einer "organischen und anorganischen Präparatesammlung“, Meß- und Analysengeräten auch eine kleine Bibliothek, bestehend aus Nachschlage- und Tabellenwerken. Welche der angegebenen Positionen tatsächlich angeschafft wurden, ist nicht überliefert. Ob und wann es zur Einrichtung einer physiologischen Abteilung auf Helgoland kam, konnte ebenfalls nicht ermittelt werden. Sehr wahrscheinlich ist, daß die Arbeitsmöglichkeiten auf dem Gebiet der Physiologie aus zwei Gründen nicht ausgebaut wurden: erstens wegen der chronischen Geldknappheit des preußischen Staates während der Inflation und zweitens, weil sich - einem wissenschaftlichen Trend folgend - Meeresbiologie und Physiologie damals voneinander entfernten.

Die Arbeitsmöglichkeiten - sowohl für Mitarbeiter als auch für Besucher - verbesserten sich ab 1934 auf allen Gebieten wesentlich. Mit dem neuen Gebäude der Anstalt waren jetzt nicht nur alle Voraussetzungen für physiologische, rein chemische und hydrographische Arbeiten gegeben, sondern auch für bakteriologische. Jetzt waren auch Arbeitsplätze für ornithologische Arbeiten vorhanden. So konnten zehn Ornithologen den neu mit Fangreusen ausgestatteten Garten und das ab 1926 auf dem Oberland zur Verfügung stehende ehemalige Wohngebäude der Marine nutzen. Ein besonders errichtetes Vogelhäuschen diente der Haltung und Beobachtung verschiedener Zugvögel. Wichtig war, daß es beheizbar war und deshalb auch im Winter Forscher darin arbeiten konnten.

Darüber, auf welchen Gebieten gearbeitet wurde, existieren nur lückenhafte Angaben. Es ist wahrscheinlich, daß die meisten Besucher Zoologen waren. Über das Jahr 1895 ist bekannt, daß zwölf Zoologen und zwei Botaniker kamen. Im Jahr 1929 arbeiteten 43 Gelehrte auf dem Gebiet der Zoologie, vier zu tierphysiologischen Themen, zehn auf dem Gebiet der Botanik, einer auf dem der Meteorologie, einer als Geologe.

Die Mehrzahl der Tischnutzer waren Deutsche, einige aber auch Amerikaner und Engländer, es blieben jedoch stets weniger Ausländer als in der Zoologischen Station Neapel. Neben den Nutzern der Arbeitstische kamen auch Gäste aus dem Ausland, die sich über den Stationsbetrieb informieren wollten. Bekanntlich war etwa ab 1900 insbesondere von amerikanischer Seite das Interesse an deutscher Wissenschaftsorganisation groß. Man 
wollte eigene Institute (z.B. innerhalb der Rockefeller-Foundation) aufbauen und war bestrebt, von Deutschland zu lernen. So besuchte im Jahre 1908 der Algologe und Protozoologe Charles Kofoid ${ }^{162}$ bei einer Informationsreise durch Europa auch die Biologische Anstalt Helgoland. Er wirkte von 1904-1936 als Professor für Zoologie in Berkeley. Aus seinen Briefen an Kollegen und dem 1910 veröffentlichten Buch „The Biological Stations of Europe" geht hervor, daß er viele Anregungen für den Aufbau einer biologischen Station in den USA bekam.

Leider sind keine geschlossenen Angaben darüber überliefert, welche Wissenschaftler und Studenten auf Helgoland arbeiteten, Informationen wurden zufällig in Publikationen gefunden. So arbeitete 1911 der schon erwähnte Otto Meyerhof auf Helgoland, die Professorin Rhoda Erdmann ${ }^{163}$, eine anerkannte Kapazität auf dem Gebiet der experimentellen Zellforschung, führte hier ebenfalls Untersuchungen durch. Die auf Helgoland entstandenen Arbeiten von Dr. Margarete Zuelzer über Spirochaeten, einzellige Mikroorganismen, fanden Eingang in das zehnbändige Standardwerk „Tierwelt der Nord- und Ostsee“. Des weiteren arbeitete der Physiologe Ernst Ziegler ${ }^{164}$ von Anfang August bis Mitte September an der Anstalt. Ziegler gehörte zu den Schülern Weismanns und beschäftigte sich mit vergleichender Entwicklungsgeschichte (vgl. M. Lücke 1985). Er schrieb u. a. ein „Lehrbuch der vergleichenden Entwicklungsgeschichte der niederen Wirbeltiere“ (1902), außerdem gab er die Sammlung „Natur und Staat, Beiträge zur naturwissenschaftlichen Gesellschaftslehre" in zehn Bänden heraus (1903-1918) sowie ein zoologisches Wörterbuch.

Zu den Gästen, die des öfteren auf Helgoland forschten, gehörte auch der Zoologe Johannes Daniel Meisenheimer ${ }^{165}$. Meisenheimer arbeitete über Probleme der Entwicklungsgeschichte und Vererbung, so wurde er als Verfasser einer zweibändigen „Entwicklungsgeschichte der Tiere“ und einer „Vererbungslehre“ (1923) bekannt. Auch sein Leipziger Kollege, der Botaniker Wilhelm Ruhland ${ }^{166}$, nutzte gern die Forschungsmöglichkeiten auf Helgoland. Wilhelm Ruhland, berühmt geworden als Herausgeber des Handbuchs der Pflanzenphysiologie, war 1922 als direkter Nachfolger Friedrich Johannes Czapeks ${ }^{167}$ auf den Lehrstuhl von Wilhelm Pfeffer ${ }^{168}$ in Leipzig berufen worden. Zunächst hatte er sich mit anatomischen, ökologischen, entwicklungsgeschichtlichen, floristischen und pflanzengeographischen Problemen befaßt, ehe er über die Phytopathologie zur Physiologie gelangte. Sein Hauptarbeitsgebiet war die Zell- und Stoffwechselphysiologie der Pflanzen. Insbesondere interessierten ihn die Permeabilität von Zellen, aber auch Probleme des Zuckerstoffwechsels. Zusammen mit Fachkollegen gab er die Zeitschriften „Planta“ und „Ergebnisse der Biologie“ heraus sowie "Monographien aus dem Gesamtgebiet der Physiologie der Pflanzen und Tiere“. Er, der sehr vielseitig war und die Botanik stets als „Gesamtgebiet“ betrachtete, hatte eine Vielzahl von Schülern, die er auch durch Vermittlung von Forschungs- und Studienaufenthalten auf Helgoland förderte. Sowohl er als auch Meisenheimer engagierten sich auch nach dem I. Weltkrieg beim Kultusministerium von Sachsen dafür, daß das Land trotz größter finanzieller Schwierigkeiten weiterhin Arbeitstische mietete. Einheimischen Studenten und Wissenschaftlern fiel es auf Grund der gestiegenen

162 Charles Kofoid (1865-1947)

163 Rhoda Erdmann (1870-1935)

164 Ernst Ziegler (1858-1925)

165 Johannes Meisenheimer (1873-1933)

166 Wilhelm Ruhland (1878-1960)

167 Friedrich Czapek (1868-1921)

168 Wilhelm Pfeffer (1845-1920) 




Abb. 41. Die Cytologin Rhoda Erdmann (1870-1935) weilte nach dem I. Weltkrieg zu einem Arbeitsaufenthalt auf Helgoland. Foto aus Revue Cibi Heft 19, 1941, Bale, S. 649

Lebenshaltungskosten immer schwerer, Arbeitsplätze zu besetzen. Einige wenige Namen sind aus Unterlagen der Leipziger Universität überliefert. So schickte Meisenheimer regelmäßig Studenten der Biologie bzw. der Zoologie nach Helgoland, darunter 1923 Friedrich Brock, 1926 Hans Kummerloeve ${ }^{169}$ und Heinz Graupner ${ }^{170}$. Kummerloeve übernahm ab Wintersemester 1936 die Vertretung für den Lehrstuhl Zoologie an der Technischen Hochschule Dresden, wo ihm 1937 die Leitung des Zoologischen Instituts übertragen wurde. 1926 war Meisenheimer auch selbst auf der Insel. Heinz Graupner weilte 1928 noch einmal auf Helgoland. 1924 kam der Dresdner Gottfried Fichtner, 1927 Wilhelm Ludwig ${ }^{171}$ und 1928 noch einmal Graupner. Einen Arbeitstisch des Jahres 1929 besetzten auch Friedrich Beckert ${ }^{172}$ aus Leipzig, 1932 Harald Fried ${ }^{173}$ und Ingo Krumbiegel ${ }^{174}$. Von Krumbiegel ist bekannt, daß er später den Zoologischen Garten in Dresden als Direktor übernahm. Über die Arbeitsthemen der Studenten ist leider nichts bekannt, von wenigen Ausnahmen abgesehen. So arbeitete Curt Hoffmann ${ }^{175}$, ein Schüler Ruhlands, 1925 über Stoffaufnahme

169 Hans Kummerloeve (geb. 1903), hieß vor 1945 Kummerlöwe

170 Heinz Graupner (geb. 1906)

171 Wilhelm Ludwig (1901-1959)

172 Friedrich Beckert (geb. 1903)

173 Harald Fried (geb. 1909)

174 Ingo Krumbiegel (geb. 1903)

175 Curt Hoffmann (1898-1959) 
an Meeresalgen. Hoffmann wurde um 1925 Assistent in Kiel und wurde 1937 als Diätendozent mit der Leitung der Botanischen Abteilung des im selben Jahr eröffneten Instituts für Meereskunde betraut. Dort wirkte er ab 1946 als außerordentlicher Professor und ab 1956 als Ordinarius für Meeresbotanik. Auch Heinrich Prell ${ }^{176}$, ordentlicher Professor an der Forstlichen Hochschule Tharandt, und Friedrich Alverdes ${ }^{177}$ aus Halle beschäftigten sich 1925 mit botanischen Themen. Prell und Alverdes fanden so viel Interesse an der Arbeit auf Helgoland, daß sie ab 1921 die Leitung eines meeresbiologischen Kurses an der Anstalt übernahmen (s. 5.2.).

Professor Willi Kuhl ${ }^{178}$, der Leiter des Instituts für Kinematische Zellforschung der Universität in Frankfurt, hatte Anfang der zwanziger Jahre bereits das Material für seine Habilitation auf Helgoland gesammelt. Insgesamt war er 27 Jahre lang regelmäßig Gast der Biologischen Anstalt und analysierte, später zusammen mit seiner Frau Gertrud, die Entwicklung und Bewegungsphänomene verschiedener Meeresorganismen mit den Methoden der Zeitraffung und -dehnung. 12 Filme stehen bis heute für den Unterricht an Hochschulen zur Verfügung. Nach dem Tode Willi Kuhls wurde ein Gastlabor, welches weiterhin der kinematischen Forschung dienen sollte, nach ihm benannt.

Die Gastwissenschaftler auf Helgoland waren bis 1930 vorwiegend Männer, von Rhoda Erdmann und Margarete Zuelzer abgesehen. Ansonsten war es für Frauen wohl sehr viel schwerer, einen Arbeitsplatz zu erhalten, insbesondere dann, wenn sie sich noch nicht durch wissenschaftliche Leistungen ausgewiesen hatten. 1930 drang eine weitere Wissenschaftlerin in die Männerdomäne ein. Um „Fräulein Ulbricht“ für den Kurs anzumelden, mußte Meisenheimer eine ausführlichere Begründung liefern, als für männliche Kollegen üblich war. Er bescheinigte Fräulein Ulbricht, daß sie durchaus über genügend Vorkenntnisse verfüge, um mit wirklichem Vorteil diesen Arbeitsplatz zu nutzen. Zwei Jahre später folgte ihr eine weitere Frau, Ilse Stempel ${ }^{179}$.

\subsection{Die Durchführung von Kursen}

Die ersten Kurse, die auf der Insel durchgeführt wurden, waren ab 1900 solche für Staatsbeamte. Ab Mai 1900 gab es einen Unterweisungskursus für Fischereibeamte, an dem acht hohe Regierungsbeamte bzw. -Assessoren und ein Oberfischmeister teilnahmen. Über das Kursprogramm für Offiziere und Staatsbeamte ist wenig bekannt, nur soviel, daß die Professoren Heincke und Ehrenbaum Vorträge zu allgemeinen Grundzügen der Fischereiwirtschaft hielten. Bald wurden auch Kurse für Marineoffiziere veranstaltet. Das Oberkommando der Marine hatte im Rahmen des Ausbaus der Insel zur Festung angeregt, die dort stationierten Marineoffiziere zu schulen. Es war eine Übereinkunft zum gegenseitigen Vorteil, wie es hieß. Die Fischer von Helgoland erhofften von der Marine Schutz, die Anstalt neues, wertvolles Untersuchungsmaterial. Für die Marine bot sich die Möglichkeit, ihr Personal auf einer so abgelegenen Insel sinnvoll zu beschäftigen. Die Offiziere erlernten einfache Methoden der Konservierung und wurden in Ziele und Arbeitsgebiete der biologischen Forschung eingeweiht.

Heincke nutzte die Chance, in den Kursen seine wissenschaftlichen Anliegen zu propagieren. Sein pädagogisches Talent und seine Begeisterungsfähigkeit trugen dazu bei,

176 Heinrich Prell (1888-1962)

177 Friedrich Alverdes (1889-1952)

178 Willi Kuhl (1892-1972)

179 Ilse Stempel (geb. 1908) 
Menschen für die Meeresforschung zu gewinnen. So schlossen sich der Anstalt auch freiwillige Helfer an, darunter Offiziere. Beispielsweise führte der Schiffsarzt des Fischereikreuzers „Ziethen“ ab Mai 1909 für zwei Jahre Planktonuntersuchungen durch.

Zu Heinckes Leidwesen war es zu dieser Zeit nicht möglich, den 1892 in der Denkschrift zur Anstalts-Gründung geäußerten Vorstellungen zu folgen und Kurse für Wissenschaftler und wissenschaftlich Interessierte durchzuführen. Dafür waren die Bedingungen nicht gegeben, vor allem mangelte es an einem größeren Raum. Heincke bemühte sich ab 1907 intensiv darum. Daß es nicht gelang, ärgerte ihn sehr, zumal in vergleichbaren Stationen in Frankreich, England und Norwegen bereits solche Kurse existierten. Als er 1909 den Plan für einen Neubau einreichte, hatte er auch einen Kurssaal eingeplant. Wie bereits erwähnt, wurde der Plan abgelehnt. Heincke ließ in seinen Anstrengungen nicht nach und wiederholte 1911 seine Bemühungen, indem er vierzehntägige Kurse mit acht bis zehn Praktikanten vorschlug. Besonders erbost war er darüber, daß Ernst Ziegler inzwischen auf Norderney einen privaten Ferienkurs einrichtete, obwohl dort die Möglichkeiten viel schlechter waren als auf Helgoland.

Die Raumverhältnisse verbesserten sich erst, als die Biologische Anstalt das am Hafen befindliche Marinegebäude erhielt. Vom 3. August bis 11. September 1920 wurde der erste meeresbiologische Kurs durchgeführt. Aufgabe des Kurses sollte es sein, Studierenden und Lehrern der Naturwissenschaften eine Gelegenheit zu geben, die wichtigsten Vertreter der marinen Tierwelt zu beobachten und ihre Biologie kennenzulernen. Der Kurs sollte eine Ergänzung zu den üblichen zoologischen Kursen und Praktika darstellen.



Abb. 42. Wolfgang von Buddenbrock (1884-1964) gehörte in den zwanziger Jahren zu den Referenten des Zoologischen Kurses auf Helgoland. Archiv der Biologischen Anstalt Helgoland. 
Der Unterricht bestand aus Vorträgen, Demonstrationen und praktischen Übungen. In den Vorträgen wurden neben allgemein-meeresbiologischen Problemen einzelne Tiergruppen behandelt. Auf Lehrexkursionen wurden einzelne Lebensgemeinschaften und ihre Ökologie vorgestellt sowie Studienmaterial gesammelt. Daran schlossen sich morphologische Untersuchungen an. Es wurde die Zergliederung größerer Seetiere geübt und besonderer Wert auf die Herstellung, Betrachtung und Auswertung von mikroskopischen Präparaten gelegt. In beschränktem Maße wurden auch Versuche mit lebenden Tieren durchgeführt.

Als Vortragende wurden Hans Lohmann ${ }^{180}$, erster Ordinarius für Zoologie an der Universität Hamburg und seit 1913 Direktor des Zoologischen Museums in Hamburg, gewonnen und Wolfgang von Buddenbrock-Hettersdorf ${ }^{181}$, damals 1. Assistent am Zoologischen Institut in Berlin. Beide Wissenschaftler hatten bereits 1919 auf Helgoland geweilt und gehörten zu jenen, die sich für die Förderung der physiologischen Forschung an der Biologischen Anstalt Helgoland eingesetzt hatten. Buddenbrock trug über das Fachgebiet „Niedere Bodentiere" vor, Hans Lohmann lehrte Methoden der Planktonforschung. Natürlich waren auch mehrere Mitarbeiter der Anstalt an der Lehrtätigkeit beteiligt, so ließ es sich Heincke nicht nehmen, über sein Arbeitsgebiet, die Nutzfische, zu sprechen. Wilhelm Mielck informierte über Planktonorganismen. Wie sich Hagmeier später erinnerte, bereicherte besonders Mielck das Praktikum durch sehr schöne, selbst gesuchte Objekte und gefärbte Präparate. Arthur Hagmeier wurde am vielseitigsten gefordert, er führte Demonstrationen im Museum und Aquarium durch, leitete Fahrten um die Insel und vermittelte sogar geologische und hydrographische Kenntnisse. Hugo Weigold behandelte Probleme der Vogelwarte und des Vogelzuges.

Der erste Kurs wurde als großer Erfolg eingeschätzt, und alle Beteiligten wünschten regelmäßige Wiederholungen derartiger Veranstaltungen. Sie wurden deshalb als unentbehrlich beurteilt,

„... weil der lebendige Organismus der eigentliche Gegenstand unserer Wissenschaft, nirgends in so mannigfaltigen Gestalten und in seinen Beziehungen so leicht erforscht und erkannt werden kann, wie in einer biologischen Station am Meere, wo wir den Quellen des Lebens näher sind als auf dem Lande" (28).

Um in Zukunft noch besser lehren zu können, beantragte Heincke einen großen Projektionsapparat. Damit sollten nicht nur Diapositive und mikroskopische Präparate, sondern auch vor allem größere und kleinere lebende Tiere „kinematographisch projiziert" werden. Er versprach dem Minister, mit diesem Apparat auf Helgoland in Zukunft Hervorragendes zu leisten.

Seine Bemühungen wurden vom Kultusministerium gewürdigt. Der Minister versicherte, alle von Heincke gegebenen Anregungen, die die Kurse betrafen, zu fördern. Allerdings, so schränkte er ein, ließen sich wohl in den jetzigen wirtschaftlichen Notzeiten nicht alle Pläne verwirklichen.

Der Kurs wurde von nun an wegen der großen Nachfrage jährlich zweimal durchgeführt, nämlich im August und September. Später wurde er durch andere Fächer ergänzt. Genaue Zahlen über die Anzahl der Kursteilnehmer liegen nicht vor. Belegt ist, daß viele Pädagogen teilnahmen. Vermutlich hatte dies etwas mit den nach dem I. Weltkrieg aufkommenden reformpädagogischen Strömungen in der Schulbiologie zu tun, die das Interesse an praktischer Naturerforschung förderte. So hatte der Biologieunterricht von der Arbeitsschulbewegung eine Vielzahl von Impulsen erhalten. Die sogenannten „praktischen

180 Hans Lohmann (1863-1934)

181 Wolfgang von Buddenbrock-Hettersdorf (1884-1964) 
Schülerübungen“ und „Schülerbeobachtungen“ rückten mehr und mehr in den Mittelpunkt des Biologieunterrichts. Einmütig wurde die Forderung erhoben, das Naturobjekt zur Anschauung heranzuziehen. Vom Lehrer wurde erwartet, daß er sich zur Ausführung von Versuchen befähige, um seine Schüler anleiten zu können (vgl. Karstädt 1929). An den meisten Schulen wurden Aquarien, Terrarien und naturkundliche Sammlungen eingerichtet. Solche Objekte verkaufte auch die Anstalt. Auch Schulgärten waren in Mode gekommen. So stellte die Anstalt nach dem I. Weltkrieg ihren ornithologischen Versuchsgarten in der Sapskuhle als Schulgarten zur Verfügung.

Später wurde noch ein kleiner zoologischer Kurs auswärtiger Dozenten in den Lehrbetrieb der Anstalt übernommen und ein botanisches Praktikum eingerichtet, das zunächst von dem Freiburger Ordinarius Friedrich Oltmanns, dann von Ernst Schreiber geleitet wurde. Genauere Angaben über das Kursprogramm sind aus den Jahren 1928/29 erhalten geblieben. So fand der meeresbiologische Kurs vom 2.-17. 8. 1929 statt, im Anschluß daran ein botanisches Praktikum. Der meeresbiologische Kurs wurde weiterhin von Mielck geleitet, der die Kursteilnehmer mit Grundlagen der Planktonforschung vertraut machte, auch von Buddenbrock aus Kiel arbeitete mit den Kursteilnehmern weiter über Bodentiere und Nekton. Eine Einführung in die Ökologie des Helgoländer Faunengebietes und der Deutschen Bucht gab Arthur Hagmeier. Zu dieser Einführung gehörten Exkursionen zu Fuß und mit den Fahrzeugen der Anstalt, die insgesamt acht Stunden dauerten. Eingeschlossen waren Demonstrationen am Objekt und Führungen durch das Nordseemuseum. Die Helgoländer Algenflora wurde von Ernst Schreiber vorgestellt, der im Anschluß an eine Ebbe-Exkursion Demonstrationen durchführte. Fester Bestandteil des Kursprogamms waren auch Hydrographie und Nannoplanktonforschung. Hierfür war Alfred Wulff verantwortlich, Adolf Bückmann führte in die Fischereibiologie ein.

Das Lehr- und Ausbildungsprogramm wurde spätestens ab 1929 stark erweitert. Es wurden u. a. ein zoologischer Kurs angeboten, ein algologischer und ein ornithologischer. Man zog auch zahlreiche auswärtige Dozenten als Lehrkräfte heran. Zu den Wissenschaftlern, die vom 7. 6. 1929 bis 13. 10. 1929 Führungen, Vorträge und Exkursionen leiteten, gehören der Botaniker Siegfried Veit Simon ${ }^{182}$, ab 1922 ordentlicher Professor und Kustos für Botanik in Bonn, und der Physiologe Hans Kniep ${ }^{183}$, der zunächst in Würzburg, später in Berlin wirkte. Besonders Kniep, den die Deutsche Botanische Gesellschaft 1931 mit einer Gedächtnisrede würdigte, war Helgoland sehr verbunden und arbeitete auch im Urlaub im Labor (vgl. Harder 1930). Zu nennen ist weiterhin der Arzt und Zoologe Werner Panzer ${ }^{184}$, der zunächst in Freiburg wirkte und 1937 die Leitung des Zoologischen Instituts in Danzig übernahm, sowie der Zoologe Ernst Matthes ${ }^{185}$, der gemeinsam mit seinem Lehrer Kükenthal das berühmte „Zoologische Praktikum“ verfaßte, nach dem Generationen von Studenten ausgebildet wurden.

Ab 1932 standen den Kursteilnehmern Arbeitsplätze im neuen Anstaltgebäude zur Verfügung. Die Räume waren mit Zentralheizung ausgestattet, und es war für Forscher und Studenten möglich, auch während der kalten Jahreszeit ihre Arbeiten durchzuführen. Etwa ab 1934 wurden auch zunehmend ausländische Forscher zur Ausbildung herangezogen, so der Zoologe Ragnar Spaerck ${ }^{186}$ aus Kopenhagen. Zu heute noch bekannten Kursleitern des Jahres 1934 gehören u. a. der Physiologe und Botaniker Kurt Mothes ${ }^{187}$ aus Halle,

182 Siegfried Simon (1877-1934)

183 Hans Kniep (1881-1930)

184 Werner Panzer (1901-1976)

185 Ernst Matthes (1899-1958)

186 Ragnar Spaerck (1896-1965)

187 Kurt Mothes (1900-1983) 


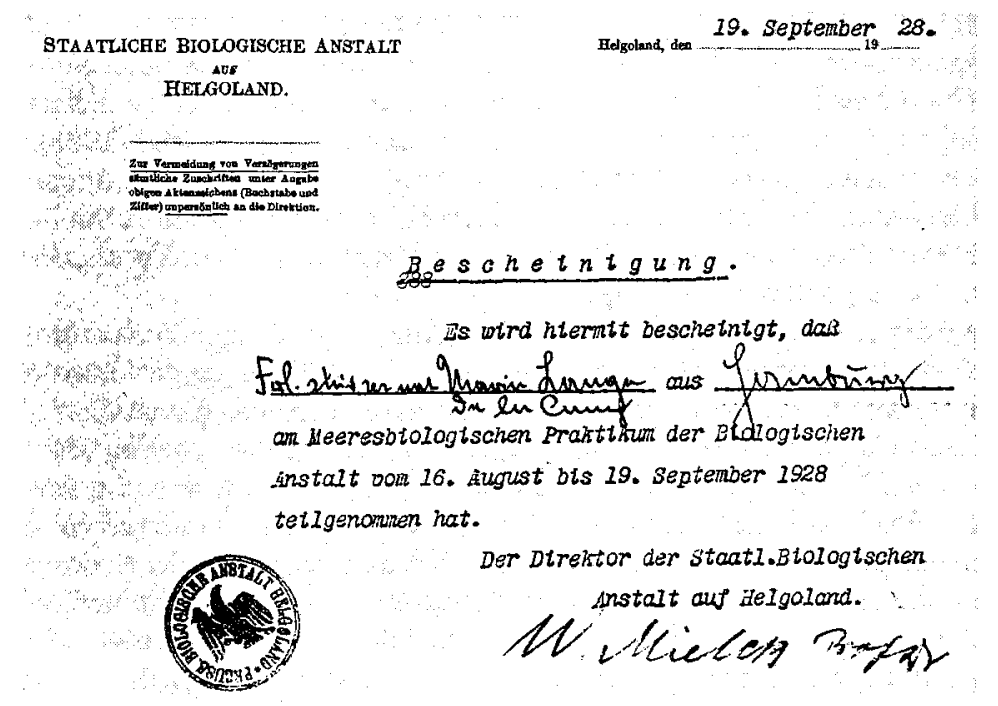

Abb. 43. „Kursschein“ aus dem Jahre 1929. Nachlaß M. Lange, Archiv der Biologischen Anstalt Helgoland.

Fritz Overbeck ${ }^{188}$ aus Frankfurt/Main, der Geograph und Meteorologe Wilhelm Meinardius $^{189}$ aus Göttingen und der Ordinarius für Geologie Ludwig Rüger ${ }^{190}$ aus Heidelberg.

Die Namen der Gelehrten, die zu den berühmtesten Deutschlands und Europas gehörten, deuten darauf hin, daß es offensichtlich als Ehre betrachtet wurde, an der Biologischen Anstalt Helgoland, der einzigen deutschen Meeresforschungsanstalt, einen Kurs zu leiten.

Besonders günstig wirkte sich hier auch die gute Beziehung Arthur Hagmeiers zur Hamburger Universität aus. Hagmeier wirkte ab 1936 als Honorarprofessor an der Mathematisch-Naturwissenschaftlichen Fakultät der Universität Hamburg. Aufgrund eines Vertrages mit der Universität wurde Studenten die Möglichkeit eingeräumt, Plätze auf Helgoland $\mathrm{zu}$ belegen. Die Biologie war inzwischen in der Schule zum sogenannten Gesinnungsund Kernfach aufgewertet worden. Eine wichtige Rolle spielte hierbei der Begriff der Lebensgemeinschaft, erstmals von Möbius an Austernbänken untersucht. Bei der politischen Ausschlachtung und Fehlbewertung der Arbeiten von Möbius und Friedrich Junge ${ }^{191}$ ging man so weit, $z u$ behaupten, daß die Organismen die Rolle einzelner Organe übernommen hätten und sich unter Negierung des einzelnen Individuums zu einem sogenannten "Organismus höherer Ordnung“ vereinigten. Dieser „Organismus höherer Ordnung“ sollte der sogenannte Volkskörper sein, die Erzeugung dieser Einsatzbereitschaft unter Leugnung der Individualität, dem sogenannten „Volksganzen“, zu dienen, war ein wesentliches Erziehungsziel. In ähnlichem Sinne äußerte sich auch der Direktor der Biologischen Anstalt Helgoland, Arthur Hagmeier, anläßlich der Einweihung des neuen Studentenheimes am 16. 8. 1934. Hagmeier sprach von fröhlicher Kameradschaft und davon, daß die jungen Leute Deutschland helfen sollten. Er erwarte von ihnen, so der Direktor, daß sie dienende Glieder des „Volksganzen“ werden und Adolf Hitler Gefolgschaft leisten würden.

188 Fritz Overbeck (1898-1983)

189 Wilhelm Meinardius (1867-1952)

190 Ludwig Rüger (1896-1955)

191 Friedrich Junge (1832-1905) 
Mit dem Studentenwohnheim standen einundzwanzig Betten zur Verfügung. Das Haus erhielt den Namen „Wilhelm-Mielck-Haus“ zu Ehren von Mielck, der durch die „Stiftung Fischkasse“ (vgl. III.) während des Weltkrieges die finanzielle Grundlage geschaffen hatte. Möglich wurde jedoch der Bau des Heimes erst durch eine großzügige Unterstützung vom Reichserziehungsministerium. Auch ein Zuschuß konnte gezahlt werden. Wie Hagmeier einschätzte, wurde es einer ganzen Anzahl von jungen Biologen durch diese Wohngelegenheit und durch den Zuschuß seitens der Anstalt möglich, sich als sogenannte „Forschungsassistenten " in die Meeresforschung einzuarbeiten und sich dann an den Forschungsaufträgen der Anstalt zu beteiligen. Der Direktor wurde im Rahmen seiner Tätigkeit an der Hamburger Universität auch zu Exkursionen eingeladen. So informierte er sein Ministerium über eine Fahrt in den englischen Kanal und beschrieb als Ziel der Unternehmung,

„Dozenten und Studenten in einer Lagergemeinschaft an Bord zusammenzuschließen und zugleich die Verbundenheit mit der Hamburgischen Universität mit dem politischen Raume in Erscheinung treten zu lassen, von dem her sich die Hamburgische Universität Sonderaufgaben in Lehre und Forschung stellt“ (58).

Zum Schluß sei erwähnt, daß nicht nur Wissenschaftler und Studenten die Studienmöglichkeiten an der Biologischen Station nutzten. Es sind Notizen über den Besuch von Malern und Bildhauern auf Helgoland überliefert. Ähnlich wie das 1902 eröffnete Aquarium in Neapel, das als erstes seiner Art besonderes Aufsehen erregte, inspirierte das Helgoländer Aquarium Künstler zur Darstellung der geheimnisvollen Meerestiere. Allerdings wurden die Kunstwerke nie so berühmt wie die in Neapel entstandenen. Paul Klee fand in Neapel Anregungen für seinen berühmten Zyklus „Die Fische“. Erinnert sei auch an seine Tagebuchnotiz über „Biester wie Polypen, Seesterne und Muscheln, schlangenartige Ungeheuer mit giftigen Augen, Riesenmaul und taschenartigem Kropf" bzw. andere, die bis über die Ohren im Sand säßen, wie die Menschheit im Vorurteil (vgl. Klee 1957).

Das Helgoländer Aquarium war fünf Jahre nach dem Neapler eröffnet worden. Mitte der zwanziger Jahre bildete Paul Flanderky in schönen Aquarellen die bizarre Unterwasserwelt ab. Vermutlich wurden die Arbeiten während des Krieges zerstört und sind nur noch durch eine Veröffentlichung überliefert worden. Der Name eines Kunstmalers, der 1929 für acht Tage hier weilte, ist nicht bekannt, ebenso nicht der jenes Bildhauers, der die Station für vier Tage besuchte. Die Bemühungen der Künstler wurden durch den Direktor unterstützt. Arthur Hagmeier liebte nicht nur die Musik, sondern auch die Malerei und förderte weitere Besuche von Künstlern. 1934 kamen zwei Kunstmaler und eine Zeichnerin. Mitte der dreißiger Jahre, etwa 1936, wurde das Treppenhaus mit Gemälden des schon erwähnten Malers Willi Ohler geschmückt. Außer Malern kamen auch sogenannte „Kinooperateure“. Diese Künstler, so hieß es ungenau, wollten sich mit wissenschaftlicher Technik vertraut machen. Besonders begehrt war die Anfertigung von Unterwasseraufnahmen im Aquarium. Das bereitete den Angestellten der Station sehr viel Arbeit, da die Fotografen die Schwierigkeiten bei der Anfertigung solcher Aufnahmen unterschätzten und nicht die nötige Geduld mitbrachten, die für das Fotografieren bizarrer mariner Organismen, z. B. von Quallen, nötig ist. Die Anstalt ging dazu über, die Aufnahmen nur noch von Fachleuten anfertigen zu lassen und die Bilder und Filme an Interessenten zu verschicken. Berühmt geworden sind die hervorragenden Aufnahmen des Helgoländer Fotografen Franz Schensky, die als Bildmappen unter dem Titel "Tier- und Pflanzenleben der Nordsee“ 1914 erstmals erschienen und dann mehrfach aufgelegt wurden. Seine Fotos fanden auch Eingang in die Biologiebücher der Schulen. 


\subsection{Ausstellung und Versand von lebenden Tieren und Präparaten}

Die Helgoländer Anstalt war nicht nur Lehrstätte, sondern auch, wie es der Kultusminister selbst ausdrückte, ein kaufmännischer Betrieb. Diese Einschätzung mag verwundern, aber durch Kurse, Versand von präparierten Seetieren, durch das Museum und das Schauaquarium konnten beträchtliche Einnahmen erzielt werden. Nachstehende Tabelle gibt einen Überblick über die Einnahmen der Biologischen Anstalt Helgoland zwischen 1892 und 1934.

Tabelle 4. Einnahmen der Biologischen Anstalt Helgoland in Mark, später Reichsmark

\begin{tabular}{|lccccc|}
\hline Jahr & Aquarium & Versand & Vermietung von Arbeitspl. & Andere & Summe \\
\hline 1892 & & 300 & & & \\
$1896-99$ & & 404 & & & \\
1909 & & 1942 & 901 & 532 & 15618 \\
1913 & 11782 & 9127 & 3483 & 3104 & 27496 \\
1924 & 13272 & 16353 & 4375 & 7372 & 41372 \\
1925 & - & 12524 & 2111 & 7177 & 21812 \\
1926 & 20425 & 17841 & 3523 & 10499 & 52288 \\
1927 & 34859 & 23791 & 4529 & 13828 & 77007 \\
1928 & 33345 & 14935 & 5439 & 9638 & 63357 \\
1929 & 28442 & 18498 & 5183 & 8115 & 60238 \\
1930 & 22806 & 10518 & 4692 & 7797 & 46323 \\
1931 & 21694 & 11317 & 4542 & 6335 & 45344 \\
1932 & 22332 & 8732 & 3143 & 5575 & 43552 \\
1933 & 25709 & 7820 & 4448 & & \\
1934 & & & & & \\
\multicolumn{7}{l}{ ab 1913} & wurden die Einnahmen des Museums und des Schauaquariums zusammen abgerech- \\
net. & & &
\end{tabular}

Die ersten Einnahmen erbrachte der Versand von Seetieren. Allerdings betrugen sie im ersten Jahr nach Stationsgründung nur 300 M. Diese Summe lag weit unter den Erwartungen, war man doch in der Denkschrift zur Anstalts-Gründung von jährlich mindestens 3000 Mark aus dem Versand lebenden und konservierten Materials ausgegangen. Heincke fühlte sich in besonderem. Maße genötigt, sich für dieses schlechte Ergebnis zu rechtfertigen. Es kam in dieser Sache zu einem längeren Briefwechsel mit Friedrich Althoff. Heincke führte das schlechte Ergebnis auf die mangelhafte Arbeit des Präparators Louis L. zurück (s. II.). Hinzu kam, daß L. beschuldigt wurde, Clemens Hartlaub durch Scheckfälschung betrogen zu haben. Die gesamte Angelegenheit wurde jedoch von seiten Heinckes mit großer Vorsicht behandelt, um das gute Verhältnis zur Familie des Angestellten nicht zu trüben. Louis L. wurde zum 1. 9. 1894 gekündigt, er siedelte nach Berlin über.

Mit dem neuen Präparator Hinrichs änderte sich die Situation, und die Anstalt konnte sich binnen weniger Jahre einen ausgezeichneten Ruf als Versenderin von Präparaten erwerben. Eine große Anzahl von Instituten forderte Material an, und es wurden damit interessante Veröffentlichungen erarbeitet. So statteten eine Reihe von Universitätsinstituten wie Berlin, Göttingen, München und Würzburg ihre neuerworbenen Seewasseraquarien mit Material von der Biologischen Anstalt Helgoland aus, außerdem die Museen Oldenburg, Marburg, Heidelberg und Breslau. Einige Besteller kannte Heincke durch langiährige Bekanntschaft, so Otto Hermes, Direktor des Aquariums in Berlin. Ebenso 
waren Exponate nach Frankfurt/Main und Hamburg geschickt worden. Auch das Bosnisch-Herzegowinische Landesmuseum in Sarajewo, das Landesmuseum und Zoologische Institut in Prag, die Botanischen Gärten in Antwerpen und Kopenhagen hatten Helgoländer Präparate erworben. Neunzig Prozent gingen an deutsche Museen und zehn Prozent ins Ausland. Darüber hinaus gehörten auch einige Privatpersonen zu den Kunden.

Die Anstalt bemühte sich sehr, auch den Wünschen und Bedürfnissen von Einzelforschern gerecht zu werden. Dies war jedoch manchmal kompliziert, da es wegen der kleinen Räume nicht möglich war, Material zu stapeln. Auch bereitete es größere Schwierigkeiten, im Winter Lebewesen aus der Tiefe zu beschaffen. Für den Versand von lebendem Material kam hinzu, daß nur zweimal wöchentlich, nämlich mittwochs und sonnabends, ein Dampfer von Helgoland abging und der Sonnabend deshalb nicht günstig war, weil die Sendungen nach ihrer Ankunft das Wochenende über auf dem Zollamt zu stehen hatten.

Nach 1910 stieg der Versand stark an. Heincke führte diese Steigerung darauf zurück, $\mathrm{da}$ von öffentlichen Aquarien und privaten Aquarienliebhabern eine sehr große Nachfrage nach lebenden Seetieren, Pflanzen und Seewasser einsetzte. Aquarien waren in europäischen Wohnzimmern in Mode gekommen. Das Interesse war so groß, daß es von den Arbeitskräften kaum noch bewältigt werden konnte. Infolgedessen wurden keine lebenden Tiere mehr ins Ausland gesandt. Auch der Versand von konserviertem Material hatte bedeutend zugenommen. Es wurde sowohl für Kurse an Universitätsinstituten verwendet als auch für Kurse, Sammlungen und als Lehrmittel für höhere Lehranstalten verwendet. So wurde z. B. 1927 eine konfektionierte Schulsammlung zusammengestellt, die allein siebzig Arten der häufigsten Tiere und Pflanzen der Nordsee, u.a. präpariert in Spiritus, umfaßte, außerdem Muschelschalen und aufgezogene Algen. Die Präparatesammlung kostete $40 \mathrm{M}$. Trotzdem gab es Wissenschaftler, die von einer wissenschaftlichen Einrichtung den kostenlosen Versand von Tieren an Wissenschaftler erwarteten. So beklagte sich der bekannte Biologe Jacob Uexküll192 1926 beim Ministerium darüber, daß er die Lieferung von Tieren, die er im Aquarium beobachten will, bezahlen müsse. Seine Beschwerde verursachte zunächst einige Aufregung; wurde jedoch abgewiesen.

Schon vor Stationsgründung hatte Heincke die Idee geäußert, ein Museum zu eröffnen. Dies entsprach nicht nur einem wissenschaftlichen Bedürfnis nach Ordnung taxonomischer Sammlungen, sondern auch dem Interesse der immer zahlreicher werdenen Badegäste. War Helgoland schon zu Beginn des 19. Jahrhunderts Wallfahrtsort für Zoologen gewesen, so entwickelte sich das 1826 gegründete Seebad (vgl. Siebs 1928) zum Erholungsort des Mittel- und Oberstandes. Kultureller Hintergrund war die gegen Ende des 19. Jahrhunderts einsetzende zweite Rückkehr zur Natur, ähnlich der Rousseauschen (JeanJacques Rousseau $\left.{ }^{193}\right)$ Naturbegeisterung, aber dem veränderten Zeitgeist gemäß praktischer gestaltet. Sie veranlaßte die Bevölkerung, freie, unverschandelte Landschaften aufzusuchen. Man reiste ins Gebirge und ans Meer. Diese neuen Neigungen fanden Niederschlag in Malerei und Literatur, es sei an Gedichte Fontanes und Engelkes erinnert. Der Naturschutz- und Tierschutzgedanke fand breite Aufnahme, was sich in Gesetzen, Vereinsgründungen usw. widerspiegelte. Erinnert sei auch daran, daß der Vogelschutz modern wurde. Für die Erhaltung einheimischer Vogelarten aus „sittlichen und wirtschaftlichen“ Gründen arbeitete seit 1896 theoretisch und praktisch besonders Hans Freiherr von Ber-

192 Jacob Uexküll (1864-1944)

193 Jean-Jacques Rousseau (1712-1778) 
lepsch ${ }^{194}$ (vgl. Gebauer 1932). Hinzu kam, daß mit dem Vertrag von Sansibar Naturbegeisterung und das Bedürfnis nach patriotischem Bekenntnis verschmolzen. Helgoland wurde zusätzlich zum Wallfahrtsort für Kriegervereine, Clubs und andere Vereinigungen.

Möglichkeiten, kulturellen Interessen auf Helgoland nachzugehen, gab es bis 1897 nur wenige. Das Interesse der Touristen, etwas über die Natur der Insel zu erfahren, war andererseits groß. Auf Helgoland existierte bereits vor Gründung der Biologischen Anstalt Helgoland eine wertvolle Präparatesammlung, deren bedeutendster Teil eine Kollektion präparierter Vögel war. Darüber hinaus hatten sich durch die Expeditionstätigkeit der Mitarbeiter der Biologischen Anstalt größere Bestände angesammelt, eine für Forschungseinrichtungen in Deutschland typische Situation, die dazu führte, daß die Zahl der Museen in Deutschland vor und um 1900 stark anstieg. Darüber, welche Aufgabe ein Museum haben sollte, wie es finanziert und organisiert werden müßte, wurde in Wissenschaftlerkreisen heftig diskutiert (vgl. Jahn 1989).

Heincke äußerte den Wunsch, daß sich die meeresbiologischen Versuchsstationen zu Sammelstätten für die „Gesamterkenntnis vom Meere" entwickeln und zum Ausgangspunkt für die Verbreitung und Fruchtbarmachung des Wissens dienen könnten. Dieses Konzept, das einen neuen Typ eines Museums hervortreten läßt, korrespondiert mit Vorstellungen der Gründer des Museums für Meereskunde am Institut für Meereskunde der Berliner Universität im Jahre 1901 (vgl. Penck 1907). Mit Hilfe des Museums, das mit der Station verbunden werden sollte, wollte Heincke Erwachsene und Kinder für die Belange der Meeresforschung interessieren.

Die Konzeption des Museums, die das Kultusministerium im Mai 1896 dem Kaiser vortrug, zielte darauf ab, sowohl eine wissenschaftliche Sammlung zu schaffen, die den Gelehrten zur Verfügung stehen sollte, als auch eine Stätte der Belehrung und Anregung für das Publikum zu begründen. Außer der naturwissenschaftlichen Sammlung sollten auch, einer früheren Idee Heinckes folgend, Sitten und Gebräuche der Helgoländer Bevölkerung vorgestellt werden. Man faßte das Anliegen sehr weit, eingebettet in das Bestreben, wie es hieß, das wiedererworbene deutsche Eiland der Bevölkerung nahezubringen. Außerdem wollte man die schon erwähnte Sammlung an einem würdigen Platz präsentieren. Die Gätkesche Sammlung stellte den Grundstock des Museums dar.

Gätke, ein Jugendgefährte Theodor Fontanes (vgl. Stresemann 1967), hatte in langjähriger Arbeit auf Helgoland eine eindrucksvolle Kollektion von Käfern, Schmetterlingen und Samenpflanzen zusammengetragen. Spezialisten wie Edmonde de Seleys-Longchamps ${ }^{195}$ und Karl-Wilhelm von Dalla Torre ${ }^{196}$ hatten sich in das reiche Material vertieft und Artenverzeichnisse angefertigt. Gätke selbst beurteilte seine Arbeit folgendermaßen:

„Durch . . . langjährige Thätigkeit als Sammler (habe ich, d. V.) auf Helgoland von Thieren und Pflanzen ein Material zusammengebracht, welches für alle ähnlichen Zielen nachgehenden Bestrebungen als gesicherter Ausgangs- und Stützpunkt bezeichnet werden kann" (64).

Besonders wertvoll war jedoch die Vogelbalgsammlung, mit der er an die lange Tradition der Vogelkunde auf Helgoland angeknüpft hatte. Sie umfaßte rund 400 auf Helgoland vorkommende Vogelarten bzw. rastende Zugvögel, darunter Seltenheiten wie die Möwe (Larus rossii). Die Vogelsammlung wollte Gätke verkaufen, um seine alleinstehenden Töchter Anna und Nina abzusichern. Darüber, wie der Ankauf zustande kam, gibt es bisher unvollständige Darstellungen, die nun (siehe Tabelle 5) ergänzt werden sollen. Gätkes Lei-

194 Hans Freiherr von Berlepsch (1857-1933)

195 Edmonde de Seleys-Longchamps (1813-1900)

196 Karl-Wilhelm von Dalla Torre (1850-1928) 
stungen in der Ornithologie waren unter deutschen Fachleuten umstritten, so gehörte Eugen Ferdinand Homeyer ${ }^{197}$ eine Zeitlang zu seinen Widersachern, außerdem Herman Schalow ${ }^{198}$. Das Manuskript von Gätkes „Die Vogelwarte Helgoland“ wurde 1886 von Schalow abgelehnt und konnte erst im Frühjahr 1891 erscheinen, weil der Ornithologe Rudolf Blasius ${ }^{199}$, Sohn des Braunschweiger Zoologen Johann Heinrich Blasius ${ }^{200}$, sich dafür verwendete.

Rudolf Blasius schrieb auch das Vorwort zu Gätkes Buch, das mit seiner überschwenglichen, deutschtümelnden Metaphorik ein eindrucksvolles Dokument des Zeitgeistes ist. Blasius schilderte die imponierende Gestalt des „Hobby“-Ornithologen, die in einem Werk Fontanes beschrieben wurde als die eines bärtigen Hünen, und stellte ihn als Beispiel dafür hin, wie ein Naturforscher zu arbeiten habe:

„In der freien Gottesnatur soll er beobachten, sich nicht allein mit der Bestimmung von toten Naturalien begnügen" (Blasius 1906).

Nach anfänglichen großen Schwierigkeiten wurde die Gätkesche Sammlung schließlich vom preußischen Staat angekauft. Die nachstehende, nach Dokumenten zusammengestellte Übersicht, faßt die Ereignisse zusammen:

Tabelle 5. Übersicht zur Geschichte des Ankaufs der Gätkeschen Vogelsammlung durch das Deutsche Reich

\begin{tabular}{|ll|}
\hline Datum & Ereignis \\
\hline August 1890 & erstes Angebot Gätkes an Kultusminister \\
Oktober 1890 & $\begin{array}{l}\text { Wiederholung des Angebots Gätkes an Kultusminister, weitere Gutachten } \\
\text { werden eingeholt, so von Möbius - Ergebnis ebenfalls positiv }\end{array}$ \\
Dezember 1890 & $\begin{array}{l}\text { Kultusministerium informiert Innenministerium, daß keine Mittel vorhanden, } \\
\text { und bittet um „Anrufung des Kaisers }\end{array}$ \\
Januar 1891 & $\begin{array}{l}\text { Gätke informiert das Kultusministerium, daß H. Seebohm }{ }^{201} \text { bereits Geld } \\
\text { geschickt hat und die Sammlung verkauft ist, nochmalige Beratung Althoffs } \\
\text { mit Möbius }\end{array}$ \\
Februar 1891 & $\begin{array}{l}\text { Reichskanzler teilt Kultusministerium mit, daß Erwerb der Sammlung durch } \\
\text { das Reich gesichert sei, Kaufvertrag werde in den nächsten Tagen } \\
\text { unterzeichnet }\end{array}$ \\
März 1891 & $\begin{array}{l}\text { Kaiserlicher Kommissar berichtet Reichskanzler, daß er die Sammlung über- } \\
\text { nommen habe, darunter 524 Vögel, 304 in Kästen und 220 einzeln, des weite- } \\
\text { ren einen Kasten mit Käfern, einen mit Schmetterlingen, eine Mappe mit Algen } \\
\text { und eine mit Phanerogamen }\end{array}$ \\
\hline
\end{tabular}

197 Eugen Homeyer (1809-1889)

198 Herman Schalow (1852-1925)

199 Rudolf Blasius (1842-1907)

200 Johann Heinrich Blasius (1809-1870)

201 Henry Seebohm (1832-1895) 
Die Bedeutung der Sammlung Gätkes wurde von vielen Fachleuten hoch geschätzt. Der englische Ornithologe Seebohm, Stahlfabrikant aus Sheffield, wollte sie sogar für das Britische Museum erwerben. Sie sollte, so Gätkes eigene Angabe, als „Gätkes Helgoland Collection“ in einem gesonderten Raum des Britischen Museums präsentiert werden. Aber ihm, so Gätkes patriotische Versicherung, sei vielmehr daran gelegen, seine Sammlung in Deutschland zu belassen. Anläßlich des Kaiserbesuchs im Jahre 1890 hatte er mit einem leitenden Staatsbeamten über seine Sammlung gesprochen und ihm sein Angebot mitgeteilt, sie an den preußischen Staat zu verkaufen. Dieser hatte ihn an das zuständige Kultusministerium verwiesen. Gätke schrieb an Gustav von Goßler ${ }^{202}$, von 1881-1891 preußischer Kultusminister:

„... meine Wünsche gehen ... dahin, daß die Sammlung für Deutschland erworben und erhalten werde, und sollten Euer Excellenz, wie ich nach den mir gemachten Mitteilungen annehmen darf, die Gründung einer zoologischen Station auf Helgoland ins Auge fassen, so würde diese der meistens geeignete Platz für die Aufgaben meiner Sammlung sein“ (64).

Einige Wissenschaftler sprachen sich in ihren Briefen an das Kultusministerium für den Ankauf der Sammlung aus, darunter Ehlers aus Göttingen und J. H. Blasius aus Braunschweig. Blasius berief sich auch auf die Autorität seines Vaters, der die Sammlung gekannt und geschätzt habe, und meinte, daß Gätke die bemerkenswerteste Kollektion zusammengetragen habe, die zwischen Petersburg und Paris existiere. Auch der Direktor der zoologischen Sammlung des Königlichen Museums für Naturkunde, Möbius, setzte sich wärmstens für den Ankauf der Kollektion ein.

Offensichtlich ließ sich der Staat aber doch mehr Zeit mit Prüfungen und Überlegungen, als Gätke recht war. Als die Sache trotz Mahnschreiben nicht vorankam, teilte er am 22. 10. 1890 dem Minister mit, daß ihm vom Britischen Museum 650 Pfund Sterling geboten worden seien (65). Am 18. 1. 1891 verkaufte er sie kurzerhand an den britischen Ornithologen Seebohm, der sie an das Britische Museum weitergeben wollte. Daraufhin wurden, wie der Übersicht zu entnehmen ist, alle Hebel in Bewegung gesetzt, um die Sammlung doch noch für Deutschland zu erhalten. Der Reichskanzler verfügte, daß aus dem Dispositionsfonds des Kaisers die erforderlichen $13000 \mathrm{M}$ zur Verfügung gestellt wurden und der Verkauf an England rückgängig gemacht wurde. Die Erhaltung der Kollektion wurde als nationale Aufgabe angesehen. Kurze Zeit, nachdem die Sammlung so mühevoll erworben worden war, wurde sie beinahe im Herbst 1891 durch einen Brand vernichtet. Dies konnte, wie aus zeitgenössischen Zeitungsberichten hervorgeht, nur durch das beherzte Eingreifen von Einwohnern und Badegästen verhindert werden. Besondere Verdienste erwarb sich hierbei der Fischhändler Hilmar Hinrich Lührs, Mitglied des Gemeinderates. Für seine mutige Tat und seine Verdienste als wissenschaftlicher Fischer wurde er mit dem Allgemeinen Ehrenzeichen geehrt. Voller Dankbarkeit schickte Lührs sein ordensgeschmücktes Selbstporträt an „seinen Kaiser“. Heinrich Gätke wurde vom Staat mit dem Titel „Professor" geehrt. Heinrich Ehlers bemerkte dazu in einem Brief an Friedrich Althoff mit viel Sinn für Realität:

„Wenn Sie für den alten Gätke den Titel ,Professor' bewirken, so wird das ein Ornament sein, über welches der alte Herr sich sehr freuen wird. Ich glaube, der Titel wird wohl von Leuten gelegentlich geführt, die ihn weniger als Gätke verdient haben“" (133).

Fünf Jahre nach Gründung der Biologischen Anstalt Helgoland konnte endlich das Museum eingeweiht werden. Für die Einrichtung hatten die Erben Nathanael Pringsheims, seine beiden Töchter Margarete und Marthe, 25000 Mark zur Verfügung gestellt.

202 Gustav von Goßler (1838-1902) 
In der Begründung der Schenkung führten die Erben aus, das Andenken ihres Vaters ehren zu wollen und die Erinnerung an seine Forschungen den späteren Generationen durch ein sichtbares Zeichen zu erhalten (99).

Das Kultusministerium beriet mit dem Zivilkabinett des Königs darüber, ob die Schenkung im Sinne der „Allerhöchsten Kabinettsorder" vom 1. 2. 1834 angenommen werden dürfe. Dies verbiete sich in dem Fall, daß Erben in ungünstigen wirtschaftlichen Verhältnissen lebten. Dies traf natürlich in diesem Fall nicht zu, Pringsheims Tochter Margarete war mit einem Geheimen Regierungsrat in Breslau verheiratet, und Tochter Marthe mit einem Rittergutsbesitzer in Zakrzervo. Man fühlte sich durch diese Schenkung auch deshalb geehrt, da Nathanael Pringsheim als einer der bedeutendsten Botaniker der Neuzeit bekannt war. Der Kaiser selbst war hocherfreut. Im Frühjahr 1896 wurde durch Kabinettsbeschluß die Einrichtung und Verwaltung des auf Helgoland zu gründenden Nordseemuseums beschlossen (100).

Der für Bau und Einrichtung erforderliche Restbetrag wurde vom Staat beigesteuert. Die Gemeinde erklärte sich daraufhin bereit, das sogenannte "Conversationshaus" gegen ein geringfügiges Eintrittsgeld zur Verfügung zu stellen. Das Gebäude wurde im Winter 1896/97 umgebaut. Im Sommer 1897 war das Museum jedoch noch immer nicht ganz fertiggestellt, wurde aber auf Druck der Bevölkerung, wie es hieß, noch Ende Juli eröffnet. Die Gemeinde wollte sich das Sommergeschäft nicht entgehen lassen. Das Nordseemuseum sollte fortan zu den Attraktionen der Insel Helgoland gehören. Vor dem Museum befand sich ein eingefriedeter Platz mit schönen Bäumen (vgl. Grieben 1908/09). Das Museum wurde Helgolandbesuchern, ebenso wie das später erbaute Aquarium, wärmstens empfohlen. Auf der Insel wurden zahlreiche Hinweisschilder und Reklamen angebracht. Das Museum war während der Badesaison vom 1.6. bis zum 1. 10. täglich von 9 bis $17 \mathrm{Uhr}$ geöffnet, während des Winters nur an einzelnen Sonntagen oder auf Anmeldung. Der Eintrittspreis betrug anfänglich generell 20 Pfennige, später an Wochentagen 50 Pfennige, an Sonntagen 20. Einheimischen und Schülern wurden Ermäßigungen gewährt. Sonderbedingungen gab es auch für Angehörige des Flottenvereins, des Kriegervereins und Mannschaften der Reichskriegsflotte.

Am 23. August 1897 fand die offizielle Feier zur Einweihung des Museums statt, die als Gedenkveranstaltung für Nathanael Pringsheim arrangiert wurde. Es nahmen etwa vierzig Personen daran teil. Die staatspolitische Bedeutung wurde durch die Anwesenheit von Regierungsvertretern, des Kommandanten der Insel, des Offizierkorps und des Gemeindevorstehers unterstrichen. Auch Wissenschaftler waren anwesend. Die Erben Pringsheims waren mit ihren Familien erschienen, darunter der bekannte Physiker Ernst Pringsheim ${ }^{203}$. Einzelheiten über die einfache, aber würdige Feier sind der Morgenausgabe der Nationalzeitung vom 24. 8. $1897 \mathrm{zu}$ entnehmen:

„Die Feier wurde . . . in dem oberen, durch geschmackvolle Netzdekorationen, Guirlanden und Blumenarrangements geschmückten Saale, vor der wohlgetroffenen Büste Pringsheims, durch das von der Badekapelle gespielte Largo von Händel eröffnet" (anonym 1899).

Die Gätkesche Sammlung war im unteren Saal aufgestellt worden. Heincke bemühte sich stets, das Museums zu erweitern. Zwischen 1898 und 1899 schaffte er einen hölzernen Sammelschrank an. Der Schrank stand an der Längswand des Saales und war etwa 14 Meter lang. Zwei ebenfalls neue hölzerne Kulissenschränke wurden zwischen den Fenstern auf der gegenüberliegenden Seite aufgestellt, des weiteren zwei durchsichtige Wandschränke aus Eisen und Glas. Ferner befanden sich im sogenannten „Vogelsaal“ ein neuer

203 Ernst Pringsheim (1859-1917) 
eiserner Doppelschrank und im Flur ein kleiner Pultschrank für geologische Sammlungen. Die vorhandenen Sammlungen wurden ständig ergänzt, so sammelte Clemens Hartlaub Meeresvögel und Enten.

Der Schwerpunkt des Museums waren die nutzbaren Tiere der Nordsee, die dem Publikum, wie es hieß, möglichst in natürlicher Umgebung vorgeführt werden sollten. Zum Zeitpunkt der Eröffnung konnte man 350 Gläser mit Präparaten präsentieren, die Tiere und Pflanzen des Meeres bei Helgoland, Fische, Hummer und Austern usw. in ihren Entwicklungsstadien darstellten. Eine besondere Zierde des Museums waren vollständige Entwicklungsserien der wichtigsten Nutzfischarten der Nordsee vom $\mathrm{Ei}$ an, die sich in keinem anderen Museum befanden. Außerdem demonstrierte man Fangmethoden der Fischerei. Eine kleine Sammlung von Schnecken, Muscheln und Algen des Meeres gab dem Besucher einen Überblick, was er am Strand finden konnte. Außerdem wurden Gesteinsbildungen und Versteinerungen von der Düne und der Insel vorgestellt. Die Gesteinssammlung wurde mit freundlicher Unterstützung des bekannten Geologen und Paläontologen Wilhelm Dames $^{204}$ zusammengestellt, der auch ein Modell der Insel entworfen hatte, das von einem Helgoländer erbaut wurde. Dieses Modell wurde gleich am Eingang dem Besucher präsentiert, damit er sich einen Überblick verschaffen konnte.

Dem Fachmann standen zwei wissenschaftliche Sammlungen zur Auswertung zur Verfügung, das von Paul Kuckuck angefertigte Algenherbar und eine Kollektion nordischer Seetiere. In den folgenden Jahren bis zum I. Weltkrieg konnte das Museum weiter ausgebaut werden. So erhielt es 1902 eine Summe von 4000 Mark, um weitere Sammlungsschränke anzukaufen. Dankbare Helgolandbesucher, so ein Frankfurter Kaufmann, stifteten Geld für den weiteren Ausbau. So konnten sowohl die wissenschaftlichen Sammlungen als auch die Ausstellung ständig erweitert werden. Nachdem im Jahre 1907 ein großer eiserner Eckwandschrank im oberen Saal des Museums für größere ausgestopfte Tiere und Skelette sowie ein eisernes Gestell zur Montage der Schmetterlingssammlung angeschafft werden konnte, war es möglich, die Sammlung publikumswirksamer zu präsentieren. Auch die ornithologische Sammlung wurde in zwei neuen Schränken untergebracht. Zu den hinzugekommenen Schauobjekten gehörten ein großer präparierter Heringshai (Lamna cornubica) und ein großes gestopftes Exemplar eines Zitterrochens (Torpedo nobiliana) aus dem nördlichen Teil der Nordsee . Eine Lehrerin aus Breslau fertigte unter Anleitung Helmuth Hertlings und Adolf Bückmanns Übersichtstafeln aus dem Arbeitsgebiet der Kommission für Meeresforschung an. Diese Darstellungen wurden ebenfalls im Museum präsentiert.

Das Nordseemuseum entwickelte sich zu einer Attraktion der Insel. 1897 besuchten 1612 Gäste das Museum, 1898 kamen 1514 und 1899 sogar 2038. Bereits 1910 konnten 19000 zahlende Besucher gezählt werden, die über 7500 Mark einbrachten.

Die Angestellten der Anstalt bemühten sich ständig, das Museum zu verschönern. So wurden im Treppenhaus Bilder von Meerestieren ausgestellt. Diese schönen, einzigartigen Autochrom-Aufnahmen von Seetieren aus dem Aquarium und der Umgebung Helgolands, die der Fotograf Franz Schenksy angefertigt hatte, wurden im Jahre 1914 durch die Anstalt angekauft.

Während das Aquarium durch die kriegsbedingten Umbauten schwer beschädigt worden war, blieb das Museumsgebäude erhalten, allerdings hatten die wertvollen Sammlungen während der Kriegszeit stark gelitten. Die Vogelbälge waren während der vier Jahre angeschimmelt und mußten nun gewaschen werden. Die seltensten Stücke wurden von nun

204 Wilhelm Dames (1843-1898) 


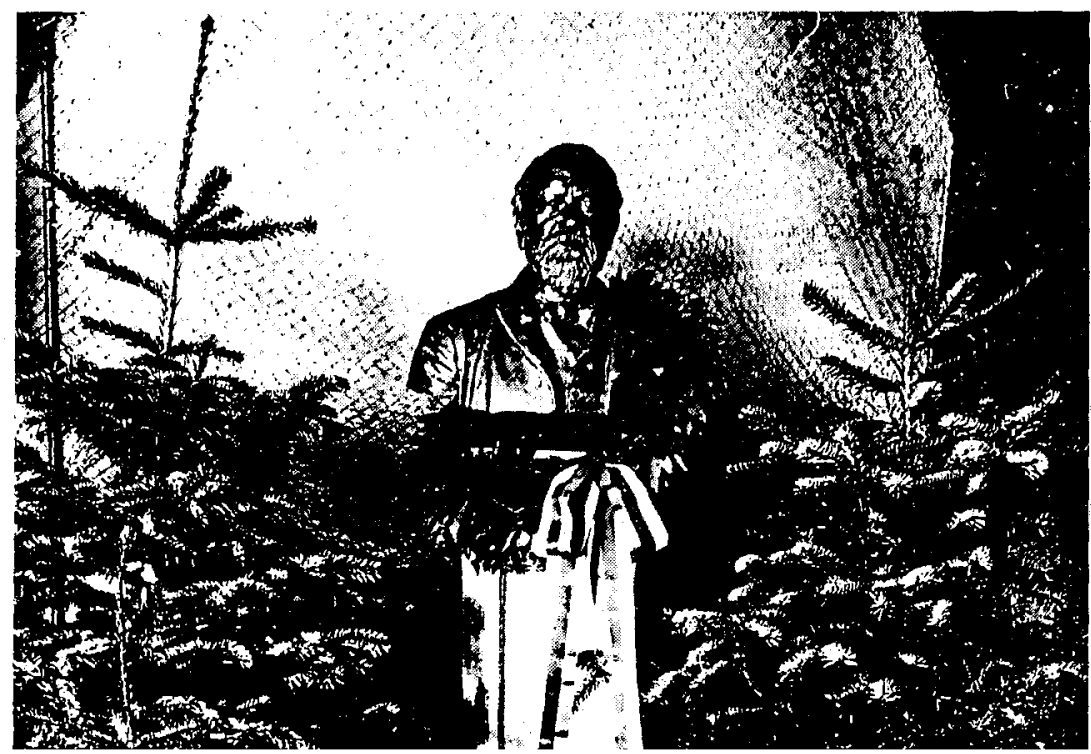

Abb. 44. Die Einweihung des Nordseemuseums mit der Büste Nathanael Pringsheims, dessen Erben Geld für den Aufbau des Museums spendeten. Archiv der Biologischen Anstalt Helgoland.



Abb. 45. Nordseemuseum. Außenansicht. Etwa 1900. Archiv der Biologischen Anstalt Helgoland. 
an in Glasröhren aufbewahrt. Die Mitarbeiter der Anstalt, besonders Hugo Weigold, leisteten Großartiges, um die Exponate zu retten.

Im Jahre 1922 wurde der Bau eines neuen Anstaltsgebäudes beantragt. In einem Flügel sollte auch ein neues Aquariengebäude untergebracht werden. Die Begründung war sehr geschickt gewählt, lief doch damals eine Propagandakampagne gegen eine echte oder scheinbare Benachteiligung deutscher Wissenschaft und Wissenschaftler. Im Jahr des Antrags wurde eine von Regierungsrat Karl Kerkhoff ${ }^{205}$ verfaßte Broschüre „Der Krieg gegen die deutsche Wissenschaft" herausgegeben und an viele wissenschaftliche Einrichtungen verteilt. Kerkhoff, ausgebildeter Naturwissenschaftler und Begründer der Zeitschrift „Forschungen und Fortschritte“, widmete sich nach 1918 in der „Reichszentrale für naturwissenschaftliche Berichterstattung " dem Wiederaufbau der deutschen Wissenschaft. Gemäß dem Zeitgeist wurde der Antrag genehmigt, allerdings wurde die Genehmigung zwei Monate später vom Finanzminister zurückgezogen. Er verwies auf die schlechte Lage der Staatsfinanzen, derzufolge sogar die Auflösung der Biologischen Anstalt Helgoland erwogen wurde.

Mielcks Bestreben war es, die Einnahmen ständig zu steigern. Er konnte durchsetzen, daß ein großer Teil der Einnahmen für den Ausbau der Anstalt verwendet werden konnte. So gelang es ihm, nur durch Einnahmen einen wissenschaftlichen Assistenten und junge technische Hilfskräfte einstellen und besolden zu können, ohne die Staatskasse in Anspruch zu nehmen. Mielck selbst unterstützte den Versand durch eine Reihe von Planktonpräparaten, verschiedene Einzeller und Larven, die er selbst aus den Fängen isolierte und bestimmte.

Mit der Verschlechterung der wirtschaftlichen Lage gab es von seiten des Finanzministeriums Bestrebungen, die Abgaben der Anstalt an den Staatshaushalt zu erhöhen, die Hälfte aller Einnahmen sollte abgeführt werden. Hier stellte sich das Kultusministerium vor die Einrichtung, und Beamte griffen helfend ein. Es kam zu einem schroffen Schriftwechsel zwischen Kultus- und Finanzministerium. Der Kultusminister begründete seinen Protest gegen die Entscheidung des Finanzministeriums so:

"Wie ich als bekannt voraussetzen darf, besitzt die Anstalt ein großes Schauaquarium und einen Handel mit lebenden und präparierten Seetieren. Sie stellte damit in gewissem Sinne einen kaufmännischen Betrieb dar. Je nach dem Besuch Helgolands durch Badegäste und Durchreisende nimmt die Zahl der Aquarienbesucher zu oder ab und ebenso das Versandgeschäft je nach Konjunktur. Aus dieser Erkenntnis müssen wie in jedem werbenden Betrieb die Ausgaben den entsprechenden Einnahmen angepaßt werden. Der Staatskasse fließen bereits $10000 \mathrm{RM}$ aus dem Versandgeschäft und $11000 \mathrm{RM}$ an Eintrittsgeldern zu. Eine weitere Erhöhung ist nicht möglich, da die Mehreinnahmen zur Deckung der Betriebskosten Verwendung finden müssen, für die im Kassenanschlag nur ein Mindestbetrag vorgesehen werden kann. Würden Mehreinnahmen, wie beabsichtigt war, zur Hälfte der Staatskasse fließen, dann würde der Betrieb der Anstalt, soweit er die genannten Zweige betrifft, lahmgelegt werden“.(116).

Während nach der Inflation sowohl die Zahl der Museumsbesucher als auch die Zahl der Kursnutzer angestiegen war und auch viele Präparate angefordert wurden, machte sich ab 1932 die schlechte wirtschaftliche Lage bemerkbar. Es kamen weniger Besucher, nur wenige Gelehrte, und auch der Versand war gering. Die Wirtschaftskrise war bald überwunden, und das Museum Helgoland und das schöne Aquarium wurde wieder von vielen Besuchern aufgesucht. Über diese Zeit sind keine Dokumente erhalten geblieben. 
Bei den Luftangriffen vom Mai 1945 wurden Museum und Aquarium stark beschädigt. Ebenso wie das Skelett des Anstaltsgebäudes fiel es erst den späteren Sprengungen zum Opfer. Damit waren die kostbaren, über mehr als fünfzig Jahre mühsam zusammengetragenen Exponate für immer verloren. Teile der Bibliothek konnten gerettet werden.

Nach der Freigabe der Insel Helgoland für den Wiederaufbau (1952) plante man, die Biologische Anstalt an ihrem alten Standort neu zu errichten. Arthur Hagmeier erlebte nur noch den Anfang der Planung, seinem Nachfolger Adolf Bückmann, 1953 zum Direktor ernannt, gelang es, die Pläne umzusetzen. 1959 konnte die Meeresstation wiedereröffnet werden. Als Biologische Anstalt Helgoland hat sich die Institution seitdem zu einem der angesehensten meeresbiologischen Forschungsinstitute in der Welt entwickelt.

\section{Dokumentenanhang}

Die nachfolgenden 11 Dokumente wurden hauptsächlich aus der umfangreichen Überlieferung dienstlichen Schriftgutes des Preußischen Kultusministeriums ausgewählt. Es handelte sich um Schriftstücke, die in Vorbereitung der Anstaltsgründung entstanden und die die Wertungen der Verfasserin unterstreichen bzw. ergänzen sollen. Sie sind als „Schreiben“ ausgewiesen. Auch private Briefe aus dem Nachlaß Althoffs mit dienstlichem Inhalt wurden einbezogen. Um sie von den offiziellen Schreiben zu unterscheiden, wurden sie als „Briefe“ gekennzeichnet.

Die Dokumente sind chronologisch geordnet. Es handelt sich um wörtliche Abschriften, die zeitgemäße Orthographie und Grammatik wurden belassen und lediglich die Datumsangaben vereinheitlicht.

Übersicht über die aufgenommenen Dokumente:

1. E.. Ehlers an Kultusminister

28. 6.1876

2. N. Pringsheim an F. Althoff

17. 7.1890

3. Kultusminister Bosse an Wilhelm II. 18. 7. 1890

4. N. Pringsheim an F. Althoff

21. 7.1890

5. Vorstand der Dt. Zoologischen

12. 12.1890

Gesellschaft an Wilhelm II.

6. V. Hensen an F. Althoff

6. 4.1891

7. A. Dohrn an F. Althoff

7. 4. 1891

8. E. Ehlers an F. Althoff

18. 4. 1891

9. F. Heincke an F. Althoff

1. 7.1891

10. Kaufvertrag zwischen dem

1. 6.1891

Kultusministerium und dem

Polizeisekretär a. D .P. Botter

11. Kultusminister an Wilhelm II. $\quad$ 12. 6.1892 